\title{
The Simple, Intuitive Model of Neural Circuits, which Memorize Data, Recognize an Image and Recall it as Imagination
}

\author{
Andrzej Brodziak ${ }^{1,2 *}$, Alicja Różyk - Myrta ${ }^{2}$ \\ ${ }^{1}$ Institute of Occupational Medicine and Environmental Health, Sosnowiec, Poland \\ ${ }^{2}$ Institute of Medical Sciences, University of Applied Sciences, Nysa, Poland
}

*Corresponding Author: Andrzej Brodziak, Institute of Occupational Medicine and Environmental Health, Sosnowiec, Poland, Email: andrzejbrodziak@wp.pl

\section{INTRODUCTION}

The purpose of this brief communication is to draw attention to the ever greater importance attributed to the theoretical model proposed once before, explaining the essence of neuronal circuits that realize imaginations, it means which recall the mental images. This is important for understanding the essence of consciousness.

This investigations upon this idea began already in 1974 [1]. The first English language paper was published in 2001 [1]. The extensive description of the model was published in 2013 [2].

The authors of the book entitled: "Awareness? It's very simple!" formulate their theory of consciousness based on this particular model [3].

The theory is consistent with the current state of knowledge, but the model is simple enough to be able to use for teaching.
The elaborated model helps explain specific processes such as imagining future situations, novel objects, and anticipated action, as well as imagination concerning oneself, which is indispensable for the sense of identity and selfawareness.

We attempt to present here this model of neural circuitry using mainly some intuitive, demonstrative figures. We think that the model presented here is helpful in teaching the basics of knowledge about the functioning of the brain.

2. Presentation OF THE Model OF Neural Circuits, WHICH Memorize DATA, RECOGNIZE AN IMAGE AND RECALL IT IN THE ABSENCE OF THE EXTERNAL ОВJЕСT

The visual imaginary and visual perceptions rely on the same neural substrate [5-8]. The basic structures are depiced on figures 1 and 2 .

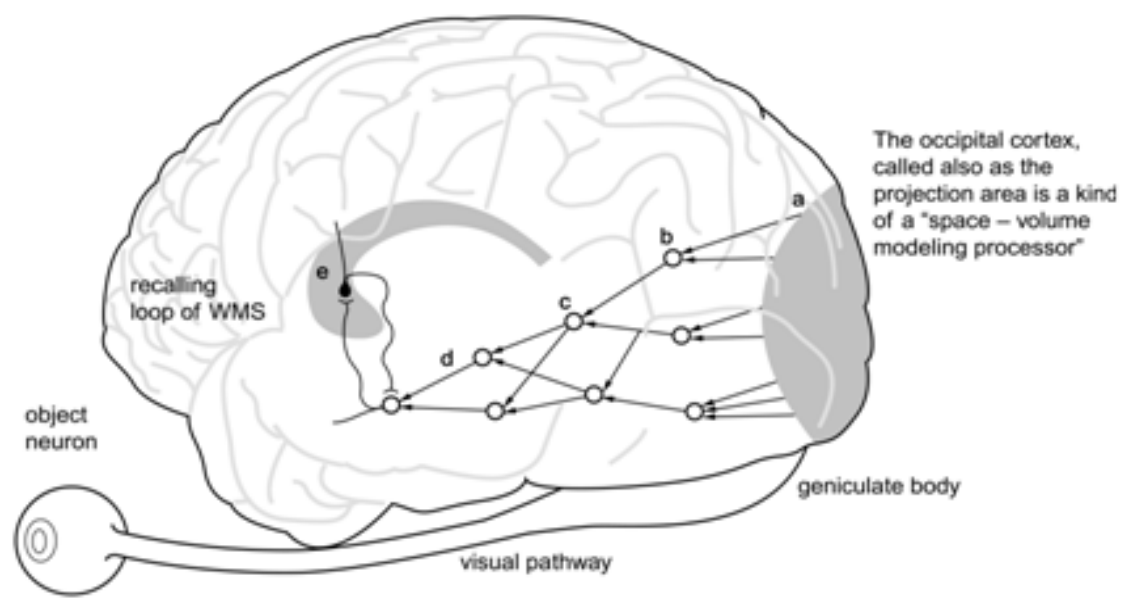

Figure1. The visual pathways does not end in the occipital region, but is prolonged by superior structures placed the anterior part of the temporal lobe, where so called 'object neuron or concept neuron' can be found. These neurons are connected with the recalling loop of the working memory system. 
The Simple, Intuitive Model of Neural Circuits, which Memorize Data, Recognize an Image and Recall it as Imagination

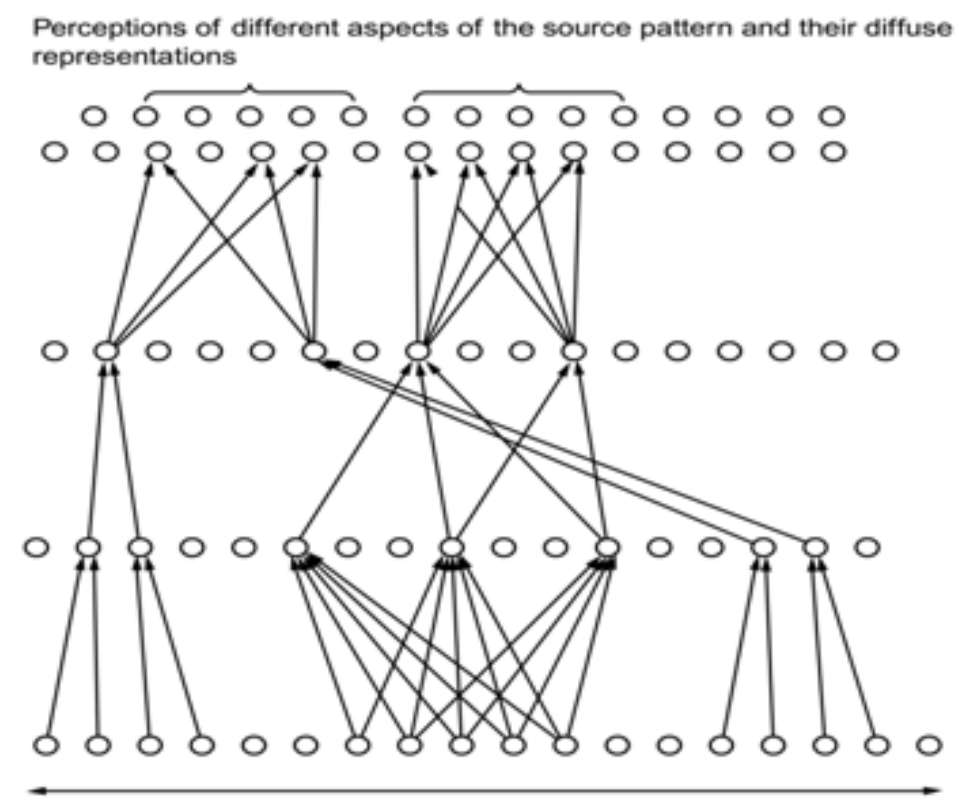

Stimuli reaching the retina (source pattern of information)

Figure2. The perceived object is represented by multiple 'object or concept neurons', which are functionally equivalent. We should realize, that apart of this multiple representation, some different aspects of the same object, such as shape, size, color or texture are perceived, as result of the parallel processing.

A very important phenomenon essential for understanding the nature of creating mental images is the oscillation between neurons of the upper and lower levels of hierarchical structures creating perceptions.

The importance of top-down pathways in creation of mental images has been recognized for several years [9]. Marcel Mesulam, in an early theoretical review paper, wrote: "Many aspects of cognition may represent an iterative neural dialogue between sensory-fugal connections, which reflect the physical nature of ambient events, and sensory-petal connections, which infer the nature of the stimulus based on empirical accounts of past experience. These reciprocal pathways, embedded within the internally generated oscillations of the brain, are further modulated by top-down projections from high-order association cortices, most prominently located in prefrontal cortex. This set of top-down projections has the capacity to transcend experience-based representations and to insert internally generated priorities into the interpretation of ongoing events" [9]. Gilbert and Siegman expressed similar opinions in their review papers, but they emphasized the role of cortico-thalamic top-down influences [10]. Fietta, in a subsequent review paper, wrote: "Top-down projections from high-order associative cortices confer the ability to transcend experience-based representations, promoting individually-sculpted interpretations, as well as mental imagery, thought, and abstraction" [11]. This is a rather metaphorical dictum. A more concrete neurophysiological sense has been accorded to the phenomenon of oscillations. Zhang stated that rhythmic neural activity exists all over the nervous system, in structures as diverse as the cerebral cortex, hippocampus, subcortical nuclei, and sense organs [13].

A more recent experimental paper published by Whitman et al. [14] pointed out that the fMRIBOLD study is able to capture states changing relatively slowly. These authors are convinced that low- and high-frequency oscillations work in concert, coordinating neural activity into whole-brain functional networks [14].

It appears that oscillations between higher level neurons and lower level neurons of hierarchical structures have an essential character. This was appreciated and carefully discussed by Lou et al. [12].

To comprehend the importance of oscillations between subcortical structures (hippocamp, thalamus) and the cerebral cortex - for teaching purposes - it is useful to consider Figures 3 and 4.

Almost all cortical neurons have recurrent axons. This was demonstrated by Carpenter's histological pictures of the cytoarchitecture of the cortex. These ramifications are necessary to start top-down pathways to activate the lower levels of the hierarchical structures at the 
moment of the stimulation of an object neuron from the side of the speech area or during complex mental processes, especially problemsolving. When the neuron of a known object is stimulated, next the activation returns - by means of recurrent axons or generally by reproductive pathways - to lower levels of the hierarchical structure, consolidated previously during perceptions and the learning process. Downward activation can even proceed to a lower projection level, such as the occipital cortex, causing vivid dreams or hallucinations.
When such a structure is activated from below by repeated perceptions, the object neuron is further stimulated by the cortex-hippocampal indexing loop. Thus, the structure of a known, recognized object is stimulated from 2 directions. When the object neuron is stimulated from the speech area, the mental image (a remembrance) is recalled. The neural mechanism of the mental imagery consists in the circulation of impulses up and down along superior levels of the hierarchical structure, which is maintained by the cortexhippocampal indexing loops (see Figures 3 and 4).

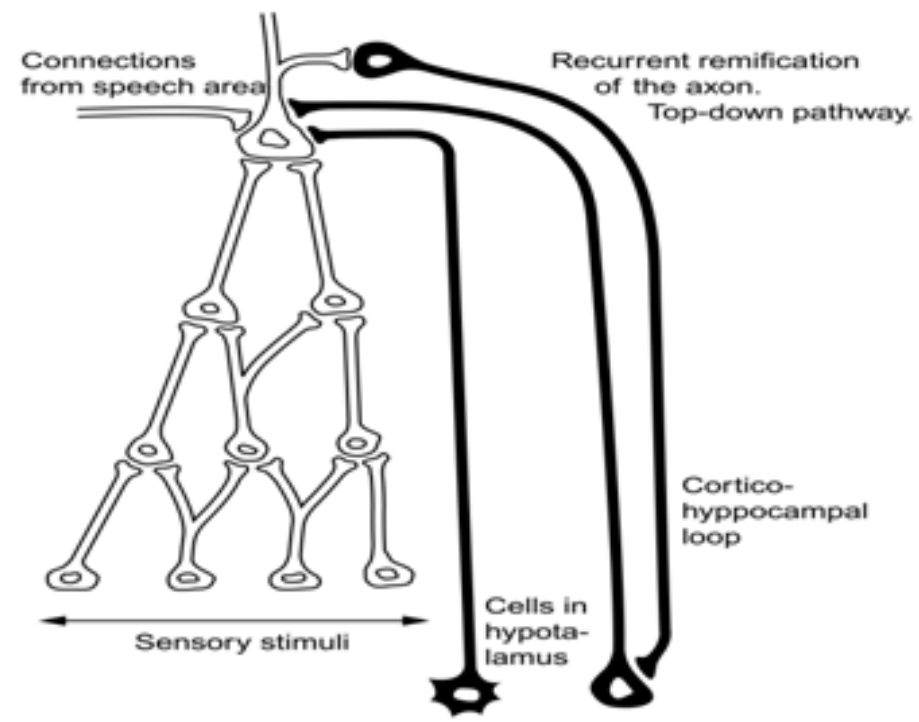

Figure3. Two kinds of connections reach so called 'object neurons'. One of them, going from the side of hypothalamus and amygdale is active in the case of emotional arousal and take part in the consolidation of the long-term memory traces. The second connection constitutes the oscillating loop, which cause that the evoked mental image is aroused for the time necessary for the action of the working memory.

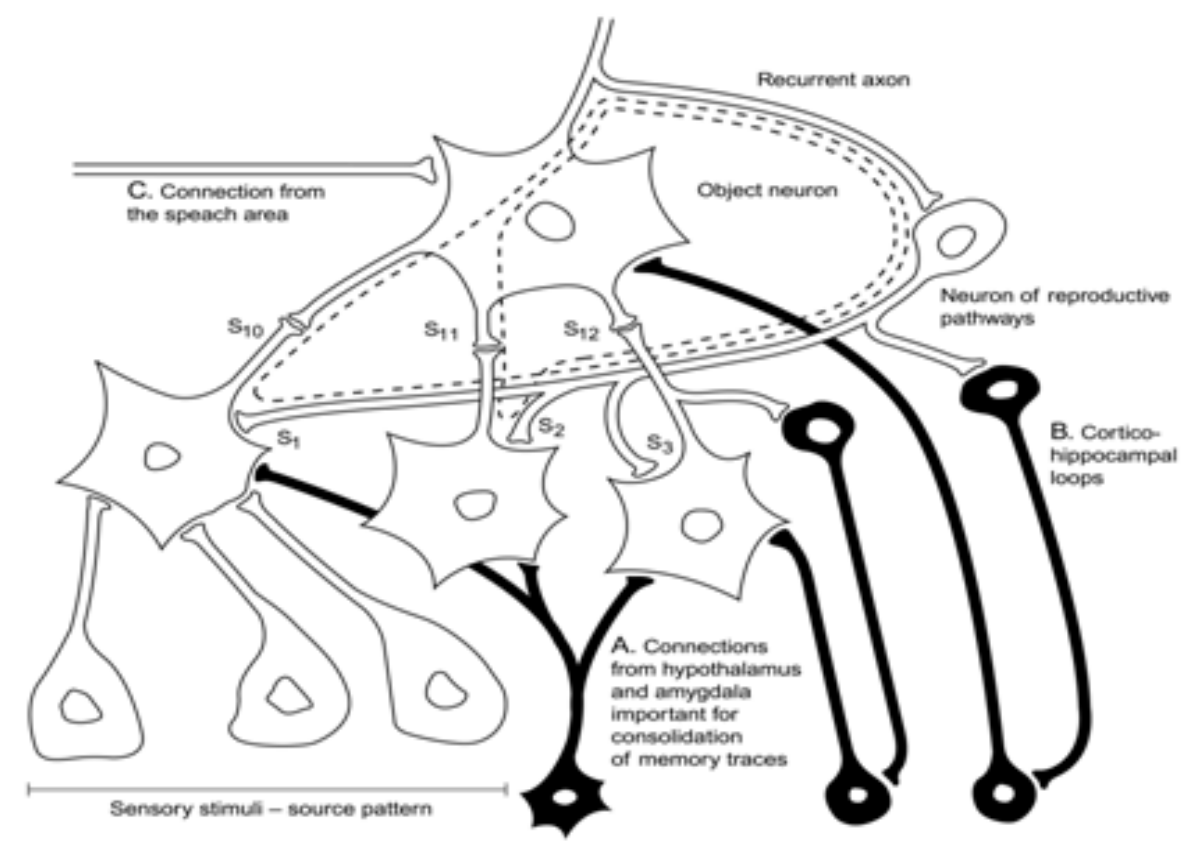

Figure4. Three layers of the hierarchical structure of neurons integrating sensory information, for instance - in the visual pathways. 
The traces of long term memory are consolidated under the influence of connections from hypothalamus and amygdale (A). There are consolidated through two mappings: weights of receiving, ascending synapses $\left(s_{10}\right.$, $\left.s_{11}, s_{12}\right)$ and synaptic weights of reproducing connections $\left(s_{1}, s_{2}, s_{3}\right)$. The second, cortico - hippocampal loop (B) is necessary for temporary recalling of the mental image. The activation of the object neuron by this indexing loop causes the recurrent reactivation of neurons in lower layers. The dotted line indicate the pathways of repetitive circulations of stimuli in the upper layers of the hierarchical structure, what is the essence of mental imaginary. The object neuron can be activated also from the side of speech area $(C)$. Mental imagery is the essence of episodic memory, short term memory and is used for working memory activity.

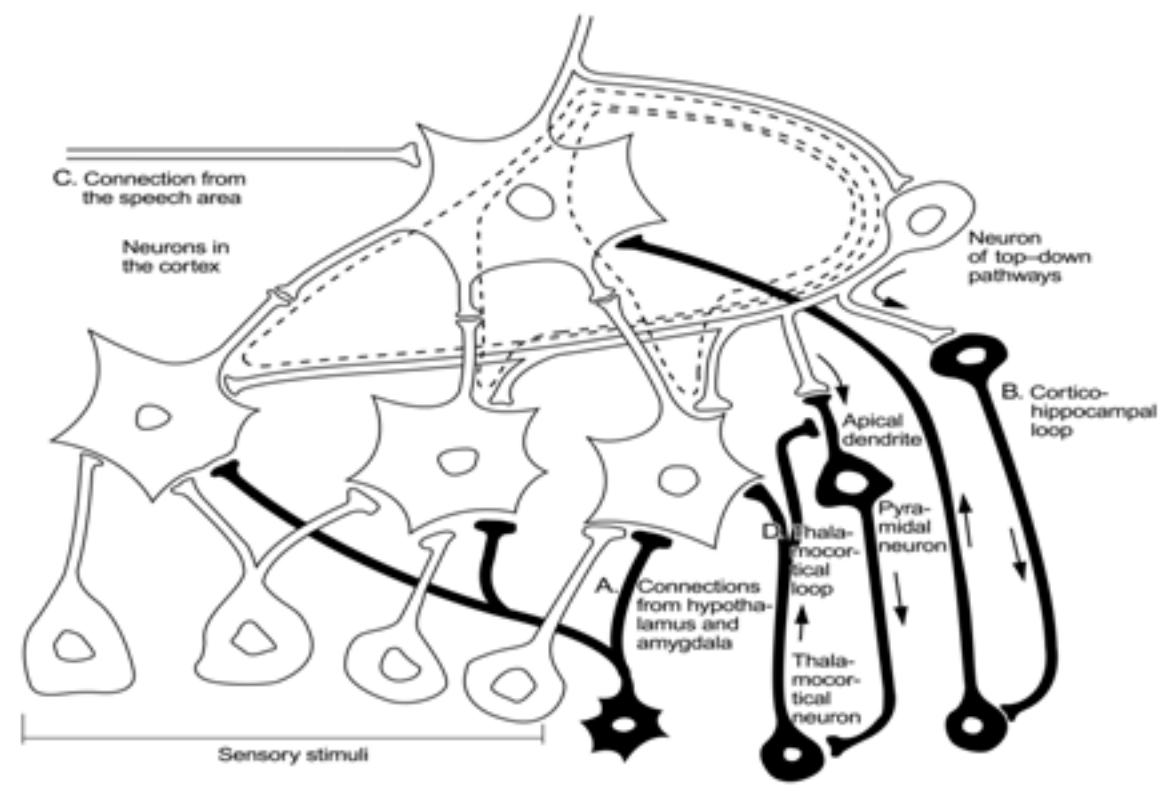

Figure5. Intuitive and symbolic depiction of some layers of the hierarchical structure of neurons integrating sensory information and of three coordinating networks.

The drawings is similar to the figure 4. It also illustrates the circuits responsible for perceptions and recalling of mental images. The significance of depicted elements are determined in the legend of the figure 4. However, here we highlight the existence of the thalamo - cortical loops. Neural activity in the period of a deep sleep is limited to the oscillations of thalamo - cortical loops reaching only the lower parts of the cortex. The frequency of these oscillations are in the theta and delta range. In this situations only a core element of the imagery process is active. The figure enable to understand why perceptions and imagery stimulate further the oscillations in these loops, what increase the frequency of oscillations. The core element of the imagery process is acting permanently without external stimuli.

It is also necessary to be aware that in the above-mentioned neuronal hierarchical structures active in recognizing objects, there are neurons that recognize situations and are active in additional aspects of perception such as spatial relations. Remembrances of a complex situation can be recalled from the memory by words such as "travel", "holidays", "harvest", "wedding", and "dancing".

The excitation of such complex imagination begins from activation of neurons located above the temporal lobes. Greenberg et al., Bartolomeo, Deselaar et al., and Huijbers et al. proved that the regions of the brain active during complex mental imagery are: the hippocampus, posterior cingulate cortex, medial, dorsolateral, and ventrolateral prefrontal cortex, angular gyrus, dorsal and ventral precuneus, anterior and mid-cingulate cortex, and supramarginal gyrus $[6-8,15]$.

\section{Significance of THE Model FOR THE UNDERSTANDING OF THE MECHANISMS UNDERLYING THE CONSCIOUSNESS}

It seems that the basic mechanism of the selfconsciousness consists on the self-excitation (reentrant processing) of neuronal circuits in cerebral subcortical structures and thalamocortical pathways. The precursors of this theory was Rodolfo Llinas, who proposed the theory of thalamo- cortical-resonance (oscillation) [16].

Georg Northoff developed this theory. According to him, one of the necessary factors for self-consciousness, is neuronal oscillations in the brainstem and midbrain [17].

So already at the begining of ontogenetic and phylogenetic development, not by reason of external stimuli from external world, but because of the structural necessity of "conscious information 
processing systems", the neural circuits are formed which triggers themselves. This is emphasized by Giulio Tononi the author of the theory of consciousness resulting from the integration of information [18, 19]. The mathematician Marcus Du Sauton, after the discussion with Giulio Tonioni, states that the basic characteristic of conscious systems is the existence of numerous asymmetric feedback connections (which is expressed or can be measured by the value of $\Phi$ parameter) [20]. Recognition of Rodolfo Llinas and Georg North off's inference as convincing opens the door to consideration of Hales's hypothesis, which assumes that secondary electric magnetic fields is possible generated on the basis of the electric pulses circulation [21].

\section{REFERENCES}

[1] Brodziak A. [ Psychonics. Theory of structures and information processes of human central nervous system and its application in computer sciences] in Polish Published by Medical Section of Polish Academy of Sciences, Katowice, 1974

[2] Brodziak A. Neurophysiology of the mental image. Med Sci Monit. 2001; 7(3):534-38.

[3] Brodziak A. A current model of neural circuitry active in forming mental images. Med Sci Monit. 2013; 19: 1146-1158.

[4] Galus W, Janusz Starzyk J. [Awareness? It's very simple!] - in Polish, ISBN: 978-83-7798-264-8, BEL Studio, Warszawa, 2018 http:// www. bel. com.pl/karta_katalogowa/items/1407.html

[5] Decety J, Jeannerod M. Imaginary and its neurological substrate. Rev Neurol. 1995; 151: 474-79.

[6] Bartolomeo P. The neural correlates of visual mental imagery: an ongoing debate. Cortex. 2008; 44:107-8.

[7] Daselaar SM, Rice HJ, Greenberg DL, et al. The spatiotemporal dynamics of autobiographical memory: neural correlates of recall, emotional intensity, and reliving. Cereb Cortex. 2008; 18: 217-29.

[8] Huijbers W, Pennartz CM, Rubin DC, Daselaar SM. Imagery and retrieval of auditory and visual information: neural correlates of successful and unsuccessful performance Neuropsychologia. 2011; 49:1730-40.

[9] Mesulam M. Representation, inference, and transcendent encoding in neurocognitive networks of the human brain. Ann Neurol. 2008; 64:367-78.

[10] Gilbert CD, Sigman M. Brain states: top-down influences in sensory processing. Neuron. 2007; 54:677-96.

[11] Fietta P, Fietta P. Cognition: neurobiological correlates and dynamics. Theor Biol Forum. 2012; 105:87-108.

[12] Lou HC, Joensson M, Biermann-Ruben K, et al. Recurrent activity in higher order, modality non-specific brain regions: A Granger causality analysis of autobiographic memory retrieval. PLoS One. 2011; 6(7):e22286.

[13] Zhang T. Neural oscillations and information flow associated with synaptic plasticity. Sheng Li Xue Bao. 2011; 63:412-22.

[14] Whitman JC, Ward LM, Woodward TS. Patterns of cortical oscillations organize neural activity into whole- brain functional networks evident in the fMRI BOLD signal. Front Hum Neurosci. 2013; 7:80.

[15] Greenberg DL, Rice HJ, Cooper JJ, et al. Coactivation of the amygdala, hippocampus and inferior frontal gyrus during autobiographical memory retrieval. Neuropsychologia. 2005; 43 : 659-74.

[16] Llinas $\mathrm{R}$ et al. The neuronal basis for consciousness. Philosophical Transactions of the Royal Society of London. Series B. 1998; 353 (1377) $1841-1849$

[17] Northoff G. What the brain's intrinsic activity can tell us about consciousness? A tridimensional view. Neurosci Biobehav Rev. 2013; 37(4):726-38.

[18] Tononi G, Koch C. The neural correlates of consciousness: an update. Annals of the New York Academy of Sciences. 2008; 1124: 239-261,

[19] Tononi G, Boly M, Massimini M, Koch C. Integrated information theory: from consciousness to its physical substrate. Nat Rev Neurosci. 2016; 17(7):450-61.

[20] Du Sauton M. The great unknown. Chapter "Consciousness", Sub chapter" The science of sleep". New York, Viking- Penguin-Random House, 2016, 339-345

[21] Hales CG. The origins of the brain's endogenous electromagnetic field and its relationship to provision of consciousness. J Integr Neurosci. 2014; 13(2):313-61.

Citation: Andrzej Brodziak, Alicja Różyk - Myrta. The Simple, Intuitive Model of Neural Circuits, which Memorize Data, Recognize an Image and Recall it as Imagination. ARC Journal of Neuroscience. 2018; 3(3):1-5. doi:dx.doi.org/10.20431/2456-057X.0303001.

Copyright: () 2018 Authors. This is an open-access article distributed under the terms of the Creative Commons Attribution License, which permits unrestricted use, distribution, and reproduction in any medium, provided the original author and source are credited. 